\title{
Doppler radar spectral width broadening due to beamwidth and wind shear
}

\author{
G. D. Nastrom* \\ Max-Planck-Institut fur Aeronomie, Katlenburg-Lindau, Germany \\ Received: 24 July 1996 / Revised: 6 January 1997 / Accepted: 30 January 1997
}

\begin{abstract}
The spectral width observed by Doppler radars can be due to several effects including the atmospheric turbulence within the radar sample volume plus effects associated with the background flow and the radar geometry and configuration. This study re-examines simple models for the effects due to finite beamwidth and vertical shear of the horizontal wind. Analytic solutions of 1- and 2-dimensional models are presented. Comparisons of the simple 2-dimensional model with numerical integrations of a 3-dimensional model with a symmetrical Gaussian beam show that the 2-dimensional model is usually adequate. The solution of the 2dimensional model gives a formula that can be applied easily to large data sets. Analysis of the analytic solutions of the 2-dimensional model for off-vertical beams reveals a term that has not been included in mathematical formulas for spectral broadening in the past. This term arises from the simultaneous effects of the changing geometry due to curvature within a finite beamwidth and the vertical wind shear. The magnitude of this effect can be comparable to that of the wellknown effects of beam-broadening and wind shear, and since it can have either algebraic sign, it can significantly reduce (or increase) the expected spectral broadening, although under typical conditions it is smaller than the beam-broadening effect. The predictions of this simple model are found to be consistent with observations from the VHF radar at White Sands Missile Range, NM.
\end{abstract}

\section{Introduction}

The width of the Doppler radar spectrum of atmospheric motions contains information about the intensity of atmospheric turbulence at scales smaller than the

\footnotetext{
* Permanent address: Department of Earth Sciences, St. Cloud State University, St. Cloud, MN 56301 USA.

email: nastrom@tigger.stcloud.msus.edu
}

radar sample volume. Atlas (1964) provides a review of the early work on and understanding of the Doppler velocity spectrum. By the time of the Atlas' review it had been established that the observed spectral width contains contributions due to processes such as wind shears across the sample volume and the effects of finite beamwidth as well as atmospheric turbulence. Before the observed spectral width can be used to infer features of the turbulence intensity the contributions from these other processes must be removed.

Several studies have provided formulae for the spectral broadening due to single effects. Hitchfeld and Dennis (1956; quoted by Atlas, 1964) give a formula for the spectral broadening due to the effect of winds tangential to a beam with finite width. Sloss and Atlas (1968) studied the broadening from shear in the crossbeam motion of the radar scatterers. Although they pose a rather general problem, allowing for the convolution of variable illumination and reflectivity functions with a specified velocity distribution and a layer of scatterers that does not necessarily fill the beam, the general formula they obtain is unwieldy and must be simplified for practical applications. Also, their formula is given for a horizontal beam; while it could easily be adapted to a vertical beam, it is not immediately appropriate for oblique beams such as used by wind profiler radars. Atlas et al. (1969) made a similar analysis of the spectral broadening due to the shear vector along the radar beam for beams of finite width. An unpublished report by Sirmans and Doviak (1973; quoted by Gossard, 1990) gives a formula for the spectral broadening due to the shear of the radial wind component in the radial direction.

Such formulae for the spectral broadening due to individual effects have been collected in reviews and texts (e.g., Gossard and Strauch, 1983; Doviak and Zrnic, 1984; Gossard, 1990). (The formulae Doviak and Zrnic, 1984, give do not explicitly include a term for beam broadening, although they later, Doviak and Zrnic, 1993, point out that beam broadening is implicitly included in their analysis through their choice of 
coordinates.) It is generally accepted that the effects are additive because the total variance of a population of Gaussian processes is the sum of the individual variances if the processes are independent (Atlas, 1964; Gossard and Strauch, 1983; Gossard, 1990). However, beam- and shear-broadening effects are not entirely independent, as discussed more later.

The most complete approach for removing the effects of wind shear and beam broadening from the Doppler spectrum has been numerical modeling of the Doppler returns from the sample volume, allowing for the weighting effects of variable illumination across the beam and possibly variable reflectivity in the volume. Such computations have been made for special geometry by Sloss and Atlas (1968), and for more general conditions appropriate to wind profiler radars by Hocking (1983a), Fukao (1988a, b), and Cohn (1995). Hocking $(1983 b, 1986,1988)$ used a full model calculation for each spectrum to account for beam broadening and wind shear effects. Hocking (1985) notes that, while a detailed theory is the best procedure for this effort, approximate formulae such as those discussed above are available for use. For climatological studies using large data sets, it is convenient to use a simple formula to account for "nonturbulence" spectral broadening effects (e.g., Fukao et al., 1994).

As mentioned, the individual formulae discussed were obtained for differing beam orientations, or differing assumptions about beamwidth, or have other differences. The present study was initially aimed at providing a simple model formula for a general framework which combines the effects of beam broadening and wind shear within the radar sample volume for arbitrary beam directions and which is derived with a single set of assumptions. This goal has been met for the general beam geometry and wind profile conditions described later. Perhaps more importantly, the general formula developed in this study reveals a term due to the simultaneous effects of beamwidth and wind shear that has not been presented before. The effects represented by this term have appeared in detailed modeling calculations (Hocking, 1983a, 1985), although a simple formula for it has not been presented. The magnitude of this term is not always negligible. The general formula for spectral broadening effects due to shear of the background winds and beam-broadening effects for arbitrary beam directions shows patterns that sometimes differ from those for the special cases given in the references cited already.

The purpose of this study is to present a simple model of the effects of wind shear and beam-broadening on Doppler spectral widths for general beam directions. In Sect. 2, analytical expressions for the spectral broadening due to beamwidth and vertical wind shear effects are developed for 1-, 2-, and 3-dimensional models of a radar beam. It is found that a relatively simple 2dimensional model captures the salient features of spectral broadening for most atmospheric conditions and radar configurations. Numerical examples are given using radar parameters typically used by wind-profiler radars (also called clear-air radars), although these results should have application to measurements from a wide range of radars. In Sect. 3 the predictions of the theory are compared with observations from the VHF wind-profiler at White Sands Missile Range (WSMR). Sect. 4 contains summary comments and concluding remarks.

\section{Analysis}

This study deals with the broadening of Doppler spectra due to uniformly varying background winds in a radar sample volume with regular (Gaussian or linear) illumination and uniform reflectivity. Actual radar beams have 3-dimensional pulse volumes with non-uniform illumination and that also may have non-uniform reflectivity within the pulse volume. The effects of nonuniform reflectivity have been considered by others (e.g., Atlas and Sloss, 1968; Atlas et al., 1969; Hocking, 1983; Fukao et al., 1988a, b) and, while they are significant in some cases, will not be included in this study. Also, the spectral broadening effects from wind shears due to gravity waves within the sample volume have been considered by others (Murphy et al., 1994; Nastrom and Eaton, 1997) and will not be included here.

The analysis is organized as follows. First, the effects of beam-broadening and shear-broadening are illustrated separately by considering a 1-dimensional beam. Next, the model for a 3-dimensional beam with Gaussian illumination function and uniform reflectivity is presented. However, since even this simplified 3-dimensional beam is too complicated to interpret easily, a 2dimensional approximation is presented and analyzed. Results from the 2-dimensional model agree well with numerical integrations of the 3-dimensional beam model.

\subsection{One-dimensional beam}

The $n^{\text {th }}$ moment of the function $v(x)$ is defined as

$\overline{v^{n}}=\frac{\int_{-\zeta}^{\zeta} v^{n}(x) W(x) \mathrm{d} x}{\int_{-\zeta}^{\zeta} W(x) \mathrm{d} x}$

where $W(x)$ is a weighting function. For radar observations, $W(x)$ depends on the intensity and geometry of the beam as well as on the reflectivity and distribution of the scatterers. The mean $\bar{v}$ is then the first moment, and the variance is defined by

$\sigma^{2}=\overline{v^{2}}-\bar{v}^{2}$

where $\overline{v^{2}}$ is the second moment of Eq. (1). For a uniform, linear function $v(x)=a+b x$ with constant $W$ it can be shown that

$\sigma^{2}=b^{2} \zeta^{2} / 3$

Equation (3) can be applied to various cases of a 1dimensional radar beam. In the case where there is a finite beamwidth but no wind shear then the velocity 
along the beam $v(\phi)=u_{o} \sin (\phi+\alpha)$ where $u_{o}$ is the horizontal wind and is a constant, $\phi$ is the angular distance from the beam axis and $\alpha$ is the zenith distance of the beam. If the half-beamwidth is $\vartheta$ and we use $-\vartheta \leq \phi \leq \vartheta$, and if $\vartheta \ll 1$ (so that $\sin \phi \approx \phi$ and $\cos \bar{\phi} \approx 1$ ) then $v \approx u_{o} \sin \alpha+\phi u_{o} \cos \alpha$. Replacing $b$ with $u_{o} \cos \alpha$ and $\zeta$ with $\vartheta$ in Eq. (3) gives

$\sigma^{2}=\left(u_{o} \cos \alpha\right)^{2} \vartheta^{2} / 3$

This simple result is remarkably similar to Eq. (6.28) as given by Atlas (1964), except that the factor in the denominator is 3 , instead of 2.76. Indeed, using the Gaussian weighting function described by Sloss and Atlas $(1968), W(\phi)=W_{o} \exp \left[-1.3816 \phi^{2} / \vartheta^{2}\right]$, and integrating Eq. (1) over $\pm \infty$ (following Atlas et al., 1969) gives 2.76 in the denominator, i.e.,

$\sigma^{2}=\left(u_{o} \cos \alpha\right)^{2} \vartheta^{2} / 2.76$

This is the same as the first term of Eq. (18) of Sloss and Atlas (1968) and is given as Eq. (4.9) in the review by Gossard (1990).

In the case where there is vertical shear of the horizontal wind and when $\phi$ is again small with $-\vartheta \leq \phi \leq \vartheta$, then $v(\phi)=\left(u_{o}+\phi(\partial u / \partial z) R \cos \alpha\right) \sin \alpha$; $R$ is the range and $\partial u / \partial z$ is the vertical shear of the horizontal wind, taken to be constant in this case. This is again a linear model and Eq. (3) gives

$\sigma^{2}=\left(\frac{\partial u}{\partial z} \sin \alpha R \cos \alpha\right)^{2} \frac{\vartheta^{2}}{3}$

This result is similar to the second term of Eq. (18) of Sloss and Atlas (1968; also given as Eq.(4.6) of Gossard, 1990), except that again the denominator is 3 instead of 2.76, and again 2.76 would obtain if a Gaussian weighting function were used.

Next consider the case of a very narrow radar beam where beam broadening effects are ignored. If the wind shear is constant along the beam then $v$ is a function only of range $r$ and we may write $v(r)=u(r) \cos \alpha=$ $\left(u_{o}+(\partial u / \partial z) r \cos \alpha\right) \sin \alpha$. Over the range $R_{o}-\Delta R / 2 \leq r \leq R_{o}+\Delta R / 2$ and with constant W (a linear pulse), (3) leads to

$\sigma^{2}=\left(\frac{\partial u \sin \alpha}{\partial z}\right)^{2}(\Delta R \cos \alpha)^{2} / 12$

This result is the same as Eq. (4.7) of Gossard (1990; which he attributes to an unpublished report by Sirmans and Doviak, 1973) for the broadening due to the shear of the radial component in the radial direction.

For general conditions, where beam-broadening and shear-broadening effects are simultaneously present, it is not sufficient to simply add the estimates of spectral broadening from Eqs. (4)-(6) although this has been the standard practice. The reasons for this limitation will become clear by considering results from the 3- and 2dimensional beam models discussed next.

\subsection{Three-dimensional beam}

The geometry of the 3-dimensional beam is shown in Fig. 1. The component of the wind along the beam at any location is

$v(r, \phi, \chi)=\vec{v} \cdot \vec{r}_{o}=\left(u_{o}+\frac{\partial u}{\partial z} \delta z\right) \sin \phi^{\prime} \cos \chi^{\prime}$

where $\vec{v}$ is the wind, $\vec{r}_{o}$ is a unit vector along the beam axis, $u_{o} \equiv u\left(R_{o}, 0,0\right)$, and where

$\delta z=r(\cos \phi \cos \alpha-\sin \phi \cos \chi \sin \alpha)-R_{o} \cos \alpha$

$\phi^{\prime}=\cos ^{-1}\{\cos \phi \cos \alpha-\sin \phi \cos \chi \sin \alpha\}$

$\chi^{\prime}=\tan ^{-1}\left\{\sin \phi \sin \chi(\sin \phi \cos \chi \cos \alpha+\cos \phi \sin \alpha)^{-1}\right\}$

Because most wind-profiler radar beams are circular, it will be assumed here that the beam has circular symmetry although this convenient simplification is not necessary. Also, although the distribution of scatterers within the sample volume may result in a nonuniform reflectivity, we will assume uniform reflectivity for convenience. In this case the weighting function is assumed to depend only on distance from the beam axis

$W(\phi)=W_{o} \exp \left[-4 \ln 2 \frac{\phi^{2}}{\vartheta^{2}}\right]$

where $\vartheta$ is the distance in radians from the center of the beam to the one-way half-power points. The mean velocity over the sample volume with range-gate size $\Delta R$ is

$\bar{v}=\frac{\int_{0}^{2 \pi} \mathrm{d} \chi \int_{0}^{\phi_{o}} \mathrm{~d} \phi \int_{R_{o}-\Delta R / 2}^{R_{o}+\Delta R / 2} \mathrm{~d} r W(\phi) v(r, \phi, \chi)}{\int_{0}^{2 \pi} \mathrm{d} \chi \int_{0}^{\phi_{o}} \mathrm{~d} \phi \int_{R_{o}-\Delta R / 2}^{R_{o}+\Delta R / 2} \mathrm{~d} r W(\phi)}$

The mean-square velocity, $\overline{v^{2}}$, is found by replacing $v$ by $v^{2}$ in (12) and the variance is found using Eq. (2). When evaluating Eq. (12), $\phi_{o}$ is taken to be sufficiently large that the exponential factor in Eq. (11) makes further contributions to the integral negligible. Note that Eq. (7) includes no small-scale fluctuations of the velocity and

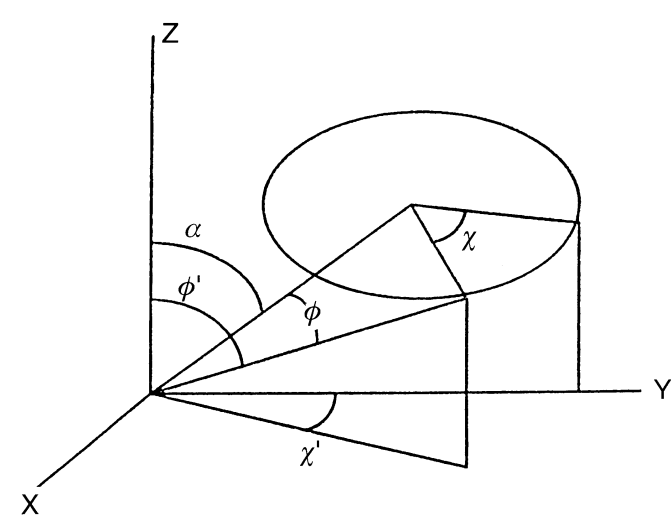

Fig. 1. Geometry of the 3-dimensional radar beam 
so the variance (the spectral broadening) here is due only to beam-broadening and wind-shear effects. Evaluation of Eq. (12), using Eqs. (7) and (11), gives the spectral broadening from these two effects for specified zenith distance, beamwidth, range, range resolution, wind shear, and background wind speed.

However, Eq. (12) is relatively complicated and simple inspection of Eq. (12) using Eqs. (7) and (11) gives little insight to the relative importance of changes in any of the variables. In an effort to gain physical understanding of the results, a 2-dimensional version of Eq. (12) will be developed and used for interpretation. It will be shown that the results from the 2-dimensional model with uniform weighting (constant $W$ ) agree very well with the results from a 3-dimensional beam with Gaussian weighting in most cases.

\subsection{Two-dimensional beam}

The geometry of the 2-dimensional beam is given in Fig. 2. In this case

$v(r, \phi)=u(r) \sin (\alpha+\phi)$

which can be expressed as

$v(r, \phi)=\left\{u_{o}+\frac{\partial u}{\partial z}\left[r \cos (\alpha+\phi)-R_{o} \cos \alpha\right]\right\} \sin (\alpha+\phi)$

where $u_{o}$ and $\partial u / \partial z$ are constants. Integration of Eq. (13) using the form analogous to Eq. (12) except in two dimensions with the limits $R_{o}-\Delta R / 2 \leq r \leq R_{o}+\Delta R / 2$ and $-\vartheta \leq \phi \leq \vartheta$ and constant $W$ gives

$\bar{v}=\frac{1}{2 \vartheta \Delta R} \int_{R_{o}-\Delta R / 2}^{R_{o}+\Delta R / 2} \mathrm{~d} r \int_{-\vartheta}^{\vartheta} \mathrm{d} \phi v(r, \phi)$

with $v(r, \phi)$ given by Eq. (13). Completing the integration for $\bar{v}$ and $\overline{v^{2}}$ and manipulating the results with the aid of standard relations for the trigonometric functions of multiple angles and the sums of angles gives

$\bar{v}=u_{o} \sin \alpha \Gamma_{1}+\frac{\partial u}{\partial z} R_{o} \cos \alpha \sin \alpha\left(\Gamma_{2}-\Gamma_{1}\right)$

and the variance, from Eq. (2), is

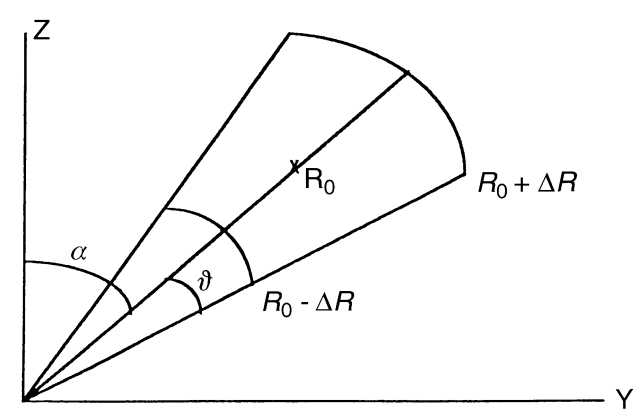

Fig. 2. Geometry of the 2-dimensional radar beam

$$
\begin{aligned}
\sigma^{2}= & \frac{u_{o}^{2}}{2}\left[1-\Gamma_{1}^{2}+\cos 2 \alpha\left(\Gamma_{1}^{2}-\Gamma_{2}\right)\right]+u_{o} \frac{\partial u}{\partial z} R_{o} \cos \alpha \\
& \times\left[\frac{3}{2} \Gamma_{1}-\Gamma_{2}-1+\frac{\Gamma_{3}}{2}+\left(2 \Gamma_{2}-\Gamma_{1}-\Gamma_{3}\right) \cos 2 \alpha\right] \\
& +\frac{1}{4}\left(\frac{\partial u}{\partial z} R_{o}\right)^{2}\left[\frac{3}{2}-\Gamma_{1}-\frac{\Gamma_{2}}{2}-\frac{\left(\Gamma_{2}-\Gamma_{1}\right)^{2}}{4}\right. \\
& +\left(1-\Gamma_{1}-\Gamma_{2}+\Gamma_{3}\right) \cos 2 \alpha \\
& \left.+\left(2 \Gamma_{3}-\Gamma_{2}-\Gamma_{4}+\frac{\left(\Gamma_{2}-\Gamma_{1}\right)^{2}}{2}\right) \frac{\cos 4 \alpha}{2}\right] \\
& +\left(\frac{\partial u}{\partial z}\right)^{2} \frac{\Delta R^{2}}{12} \frac{\left(1-\Gamma_{4} \cos 4 \alpha\right)}{8}
\end{aligned}
$$

where $\Gamma_{n}=\frac{\sin (n \vartheta)}{n \vartheta}$.

The comparisons of the results from Eq. (15) with the numerical integrations of Eq. (12) that are given in Figs. 3-5 show that Eq. (15) is a good approximation to the 3-dimensional solution, usually within a few percent. The advantage of Eq. (15) is that it is relatively straightforward to interpret physically and it can be used to study the changes in spectral broadening due to changes in wind speed, wind shear, etc. This formula also can be applied easily to large data sets where it would not be practical to integrate Eq. (12) to find the broadening effects for each entry.

The numerical values of the coefficients of the terms in Eq. (15) are given in Table 1 for the parameters of the WSMR $\operatorname{radar}\left(\alpha=15^{\circ}, \vartheta=1.5^{\circ}\right)$ and for typical ranges in the troposphere, stratosphere, and mesosphere. Clearly, each of the terms may be important for typical values of wind speed and wind shear. For example, at $17 \mathrm{~km}$ with $u_{o}=20 \mathrm{~ms}^{-1}$ and $\partial u / \partial z=0.01 \mathrm{~s}^{-1}$, the values of the terms are $0.118,-0.101,0.030$, and 0.012 , respectively. Notice that in this example the second term, that due to the product of wind speed and wind shear, largely offsets the other three terms. The physical interpretation of the individual terms is considered next.

Physical interpretation of the terms in Eq. (15) is aided by using a power series expansion (to order $\vartheta^{2}$ ) of the coefficients. Then

$$
\sigma^{2} \approx \frac{\vartheta^{2}}{3} u_{o}^{2} \cos ^{2} \alpha-\frac{2 \vartheta^{2}}{3} \sin ^{2} \alpha\left(u_{o} \frac{\partial u}{\partial z} R_{o} \cos \alpha\right)
$$

$$
\begin{aligned}
& +\frac{\vartheta^{2}}{24}(3+\cos 4 \alpha-4 \cos 2 \alpha)\left(\frac{\partial u}{\partial z}\right)^{2} R_{o}^{2} \\
& +\left(\frac{\vartheta^{2}}{3} \cos 4 \alpha+\sin ^{2} \alpha \cos ^{2} \alpha\right)\left(\frac{\partial u}{\partial z}\right)^{2} \frac{\Delta R^{2}}{12}
\end{aligned}
$$

(IV)

Note that the sign of $\alpha$ is irrelevant because $\cos n \alpha$ and $\sin ^{2} \alpha$ are symmetric with respect to $\alpha$.

All of the terms on the right hand side in Eq. (16) except term $\mathrm{V}$ (the last part of the last term) depend on 

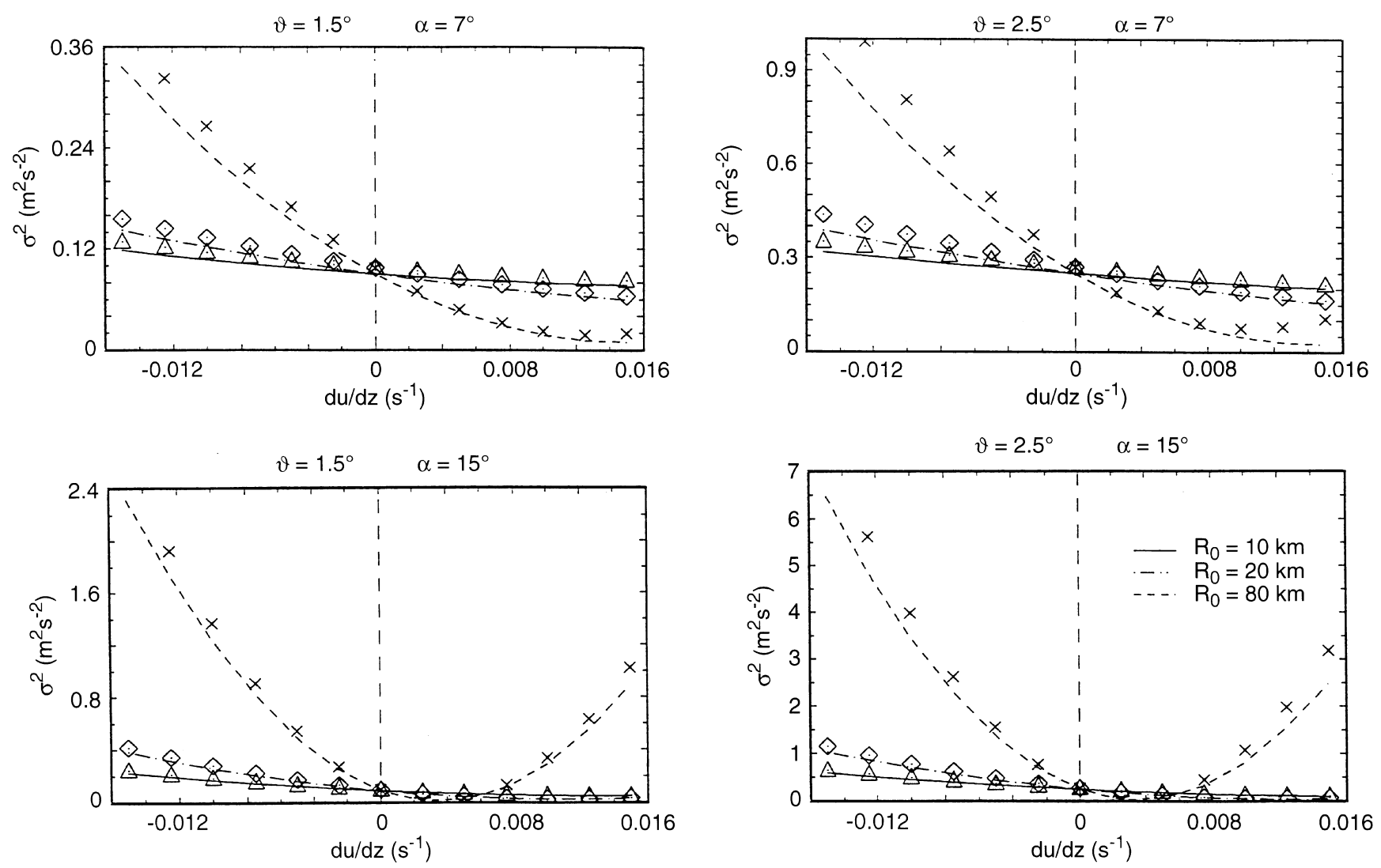

Fig. 3. Changes of the expected spectral broadening as a function of vertical shear of the horizontal wind for typical tropospheric, stratospheric, and mesospheric ranges $\left(R_{o}\right)$. Panels are for half-beamwidths of 1.5 and 2.5 degrees, and zenith distances of 7 and 15 degrees. The lines (symbols) are solutions of the 2-dimensional (3-dimensional) model. For all cases, $u_{o}=20 \mathrm{~ms}^{-1}$
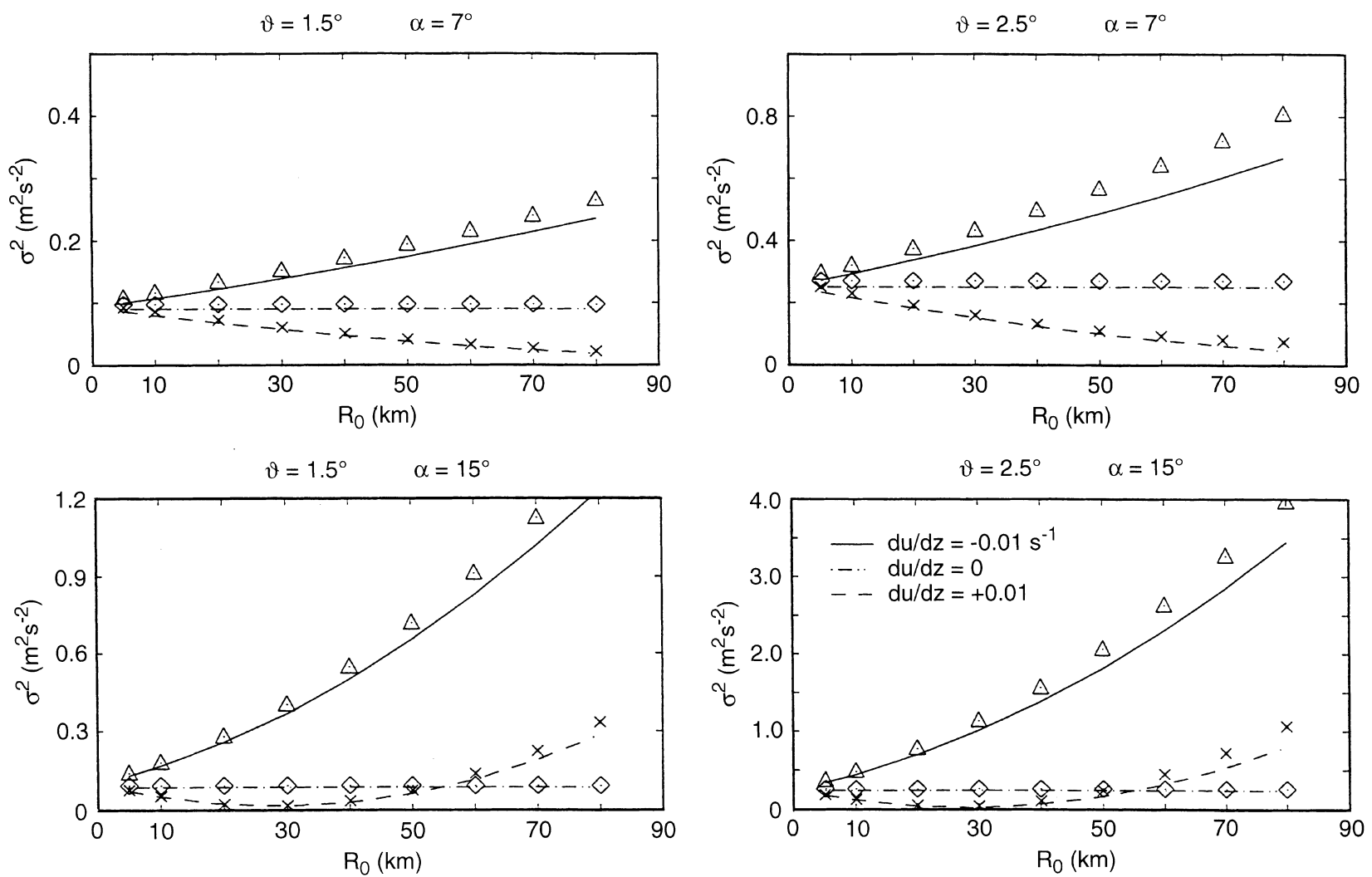

Fig. 4. As in Fig. 3, except for solutions as a function of range for typical vertical wind shear values. Note that the ordinate scales differ among panels 

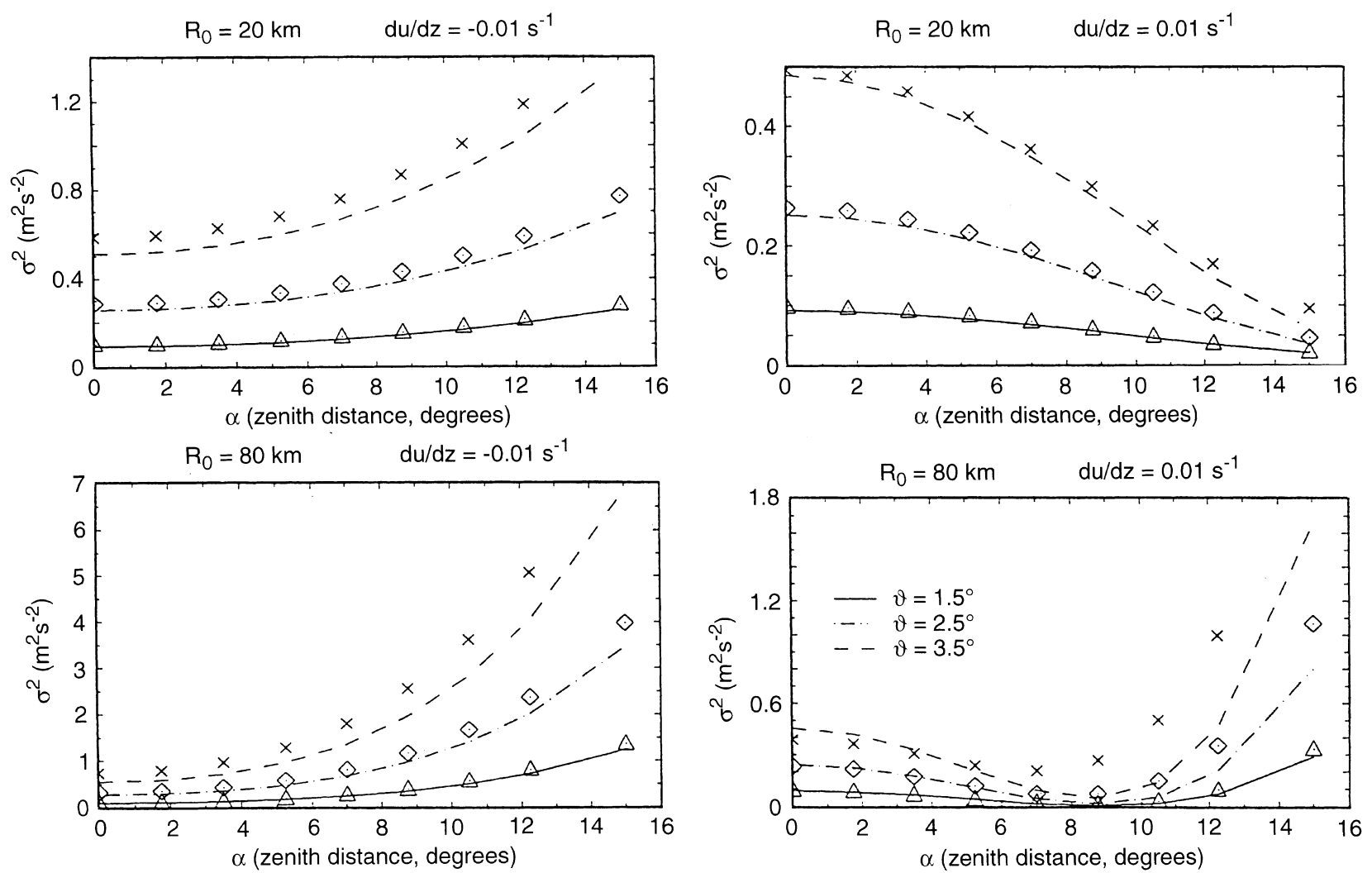

Fig. 5. As in Fig. 3, except for solutions as a function of zenith distance for typical half-beamwidths

$\vartheta^{2}$ and thus represent beam-broadening effects. The first term is due to beam-broadening effects by the background wind. If there is no vertical wind shear, then the first term is the only nonzero term and Eq. (16) reduces to Eq. (4).

The central terms, II-IV, are due to the combined effects of finite beamwidth and vertical wind shear; i.e., they arise from the wind speed changes across the beam. Terms II and III depend on the range $\left(R_{o}\right)$ as well as on the vertical wind shear and they represent the effects of the growth of the distance across the beam with distance from the radar. The sign of term II depends on the sign of the vertical wind shear and thus this term can partially offset the other terms; this term represents the effect of the changing trigonometric factor across the beam combined with the wind speed changes from vertical wind shear across the beam. Although Hocking (1985) notes that this effect has appeared in computer simulation results, a mathematical expression for term II has not appeared in past studies. As mentioned in the discussion of Table 1, this term can have significant magnitude in some cases.

Term III is roughly similar to the approximation in Eq. (5), although the magnitude of the coefficient here is much smaller for all $\alpha>0$ than that in Eq. (5) (e.g., for $\alpha=15$ degrees it is about $1 / 10$ as large). The coefficient is smaller in Eq. (16) because a curved, lens-shaped, sample volume is used here whereas in Eq. (5) (and in Sloss and Atlas, 1968) a linear approximation is used and so the sample volume is effectively like a flat plate. Note that for a typical weather radar where $\alpha \rightarrow \pi / 2$ then only terms III and IV are nonzero; term III resembles the middle term of Sloss and Atlas' Eq. (18), except with a different numerical coefficient. Term IV represents the variance due to wind speed changes simultaneously along and across the beam. Term V is from wind speed changes along the beam. It is due only to the vertical wind shear across a range gate; indeed, if the beam is very narrow so that $\vartheta^{2} \rightarrow 0$ then only term $\mathrm{V}$ is nonzero and Eq. (16) reduces to Eq. (6).

For a given experiment the beamwidth, zenith distance, and range resolution are constants and so the solutions of Eq. (15) are second order polynomials with respect to background wind speed, range, and vertical wind shear. For example, Fig. 3 shows the values of $\sigma^{2}$ as a function of vertical wind shear for $\vartheta=1.5$ and $2.5^{\circ}$, $\alpha=7$ and $15^{\circ}$, and $R_{o}=10,20$, and $80 \mathrm{~km}$ (note that the ordinate scales differ among the panels), using $u_{o}=20 \mathrm{~ms}^{-1}$ in all cases. In Fig. 3, the curves are solutions from Eq. (15) and the symbols near the curves

Table 1. Numerical coefficients of the four terms of (15) with $\alpha=1.5^{\circ}, \vartheta=1.5^{\circ}$, and $\Delta R=150 \mathrm{~m}$ (the parameters used for the WSMR radar) at typical ranges in the troposphere, stratosphere, and mesosphere. Each term is identified by its remaining variables

\begin{tabular}{lllcl}
\hline$R_{o}(\mathrm{~km})$ & $u_{o}^{2}$ & $u_{o} \partial u / \partial z$ & $(\partial u / \partial z)^{2}$ & $(\partial u / \partial z)^{2}$ \\
\hline 6 & $2.9610^{-4}$ & -0.178 & 37.4 & 117 \\
17 & $2.9610^{-4}$ & -0.504 & 301 & 117 \\
80 & $2.9610^{-4}$ & -2.37 & 6656 & 117 \\
\hline
\end{tabular}


are the 3-dimensional solutions from Eq. (12), and comparison of the curves and symbols shows that Eq. (15) is usually an adequate approximation to the 3dimensional model. It is noteworthy that, due to term II discussed already, the minima of $\sigma^{2}$ occur at nonzero wind shears.

Figure 4 shows the changes of $\sigma^{2}$ as a function of range for typical values of vertical wind shear and using the same choices of beamwidth and zenith distance as in Fig. 3. In the lower panels, for $\alpha=15^{\circ}$, note that the curves for $\mathrm{d} u / \mathrm{d} z=0.01 \mathrm{~s}^{-1}$ have minima near $30 \mathrm{~km}$ range. Differentiating Eq. (15) with respect to $R_{o}$ shows that the minimum occurs at $R_{o}=28.2 \mathrm{~km}$ when $\vartheta=2.5^{\circ}$, for example.

Extreme values of $\sigma^{2}$ with respect to any of the variables on the right hand side of Eq. (15) could be evaluated from the partial derivative, in principle. However, the dependence of $\sigma^{2}$ on zenith distance is relatively complicated and numerical results illustrate it more easily than an equation. Figure 5 shows the changes of $\sigma^{2}$ as a function of zenith distance for ranges 20 and $80 \mathrm{~km}$ and wind shears of $\pm 0.01 \mathrm{~s}^{-1}$, for $\vartheta=1.5,2.5$, and 3.5 degrees. In Fig. 5, $\sigma^{2}$ has a minimum at nonzero zenith distance when the vertical wind shear is positive, as anticipated above in the discussion of the second terms of Eqs. (15) and (16).

\subsection{Biases of mean winds and heights}

The effects of finite beamwidth and vertical shear of the horizontal wind also lead to a small bias in the mean observed wind speed, $\bar{v}$. Expanding the $\Gamma_{n}$ in Eq. (14b) in power series up to second order in $\vartheta$ gives

$\bar{v} \approx\left(u_{o}-\frac{\vartheta^{2}}{2} \frac{\partial u}{\partial z} R_{o} \cos \alpha\right) \sin \alpha$

The second term in the brackets represents the bias of the mean. The second term is negligible for most conditions in the troposphere and lower stratosphere. However, in the mesosphere the bias due to the second term may be significant; for example, the bias is about $1 \mathrm{~ms}^{-1}$ at $90 \mathrm{~km}$ range for $\vartheta=2.5^{\circ}$ and $0.01 \mathrm{~s}^{-1}$ vertical shear of the horizontal wind. The sign of the bias depends on the sign of the wind shear. The bias arises because the curvature of the sampling volume gives asymmetric height changes with respect to the beam axis and because we have assumed a constant wind shear through the lens-shaped sampling volume.

The lens-like shape of the sampling volume with uniform reflectivity causes the mean height of a vertically directed beam to be slightly less than $R_{o}$ and leads to a small amount of range-gate smearing. This effect is negligible in the lower atmosphere but may be important in the mesosphere for some applications. The magnitude of the effect can be estimated as follows. The variance from Eq. (3) of the heights within the sample volume of a vertically directed cylindrical beam with thickness $\Delta R$ is $\sigma^{2}=\Delta R^{2} / 12$. Then, using Eq. (12) to evaluate $\bar{Z}$ and $\overline{Z^{2}}$ and Eq. (2) to find $\sigma_{z}^{2}$ the effective range resolution is $\Delta R_{e f f}=\sqrt{12} \sigma_{z}$. For example, for a beam with $\vartheta=2.5^{\circ}$ and $\Delta R=150 \mathrm{~m}$, at $R_{o}=20 \mathrm{~km}$ the bias of the height $\bar{Z}-R_{o}$ and the change in effective range-resolution $\Delta R_{\text {eff }}-\Delta R$ are $-4 \mathrm{~m}$ and $0.9 \mathrm{~m}$, respectively, while at $R_{o}=90 \mathrm{~km}$ they are $-19 \mathrm{~m}$ and $17 \mathrm{~m}$.

\section{Comparisons with observations}

Figure 6 shows the hourly mean widths at WSMR at 6.1 and $17.6 \mathrm{~km}$ for the oblique beams in the east-west (beam 1) and north-south (beam 2) planes. The data used are from 1991-1995. Details of the WSMR radar system, quality control processing, and examples of the data are given in Nastrom and Eaton (1993). For this study, an hourly mean was used only if five or more observations on both beams 1 and 2 survived quality control checks at the level used and at the two levels above and below the level used. In Fig. 6, 18802 hourly means are used at $6.1 \mathrm{~km}$ and 17334 at $17.6 \mathrm{~km}$ (the range given in Fig. 6 is range from the radar, $R_{o}$, and should not be confused with height above sea-level). Vertical wind shears were computed from the differences of the averages of the winds over the two levels above and below the level used.

In Fig. 6, the straight line in each panel is the beam broadening that would be expected if only the first term of Eq. (15) were nonzero. If beam broadening were the only effect then all of the points would be expected to lie above the lines in Fig. 6 (except, of course, for the effects of observational errors in wind speed and in spectral width). Note that more points fall below the lines in the east-west beam (beam 1) at $6.1 \mathrm{~km}$ than in the other three panels. This result could be expected from Eq. (15) because both the background zonal wind speed and its vertical shear at $6.1 \mathrm{~km}$ are usually positive making the contribution from the second term of Eq. (15) negative (the coefficient of this term is seen to be negative in Table 1). At $17.6 \mathrm{~km}$ the vertical shear of the background zonal wind is negative (Nastrom and Eaton, 1995) so the second term of Eq. (15) is positive. The sign of the second term of Eq. (15) for the meridional wind component at both heights changes with synoptic conditions.

Figure 7 shows the hourly mean widths at 6.1 and $17.6 \mathrm{~km}$ plotted as a function of vertical shear of the horizontal wind. Only cases with wind speeds less than $10 \mathrm{~ms}^{-1}$ were used here. At $6.1 \mathrm{~km}$ the lower boundary of the cloud of points has a parabolic shape with a minimum value at zero shear. The value of the width at the minimum is very near the distance between spectral points at WSMR (near $0.01 \mathrm{~m}^{2} \mathrm{~s}^{-2}$ ). At $17.6 \mathrm{~km}$, on the other hand, the base of the cloud of points is nearly flat with respect to wind shear. These patterns are consistent with those expected from Eq. (15) as illustrated in Fig. 8. Figure 8 shows that there is a higher probability of finding very small values of $\sigma^{2}$ for large values of shear at $17 \mathrm{~km}$ than at $6 \mathrm{~km}$.

Another example of the effect of wind shear is illustrated in Table 2. Table 2 compares the mean spectral widths of cases observed at WSMR at $17.1 \mathrm{~km}$ sorted according to the algebraic sign of the product of 

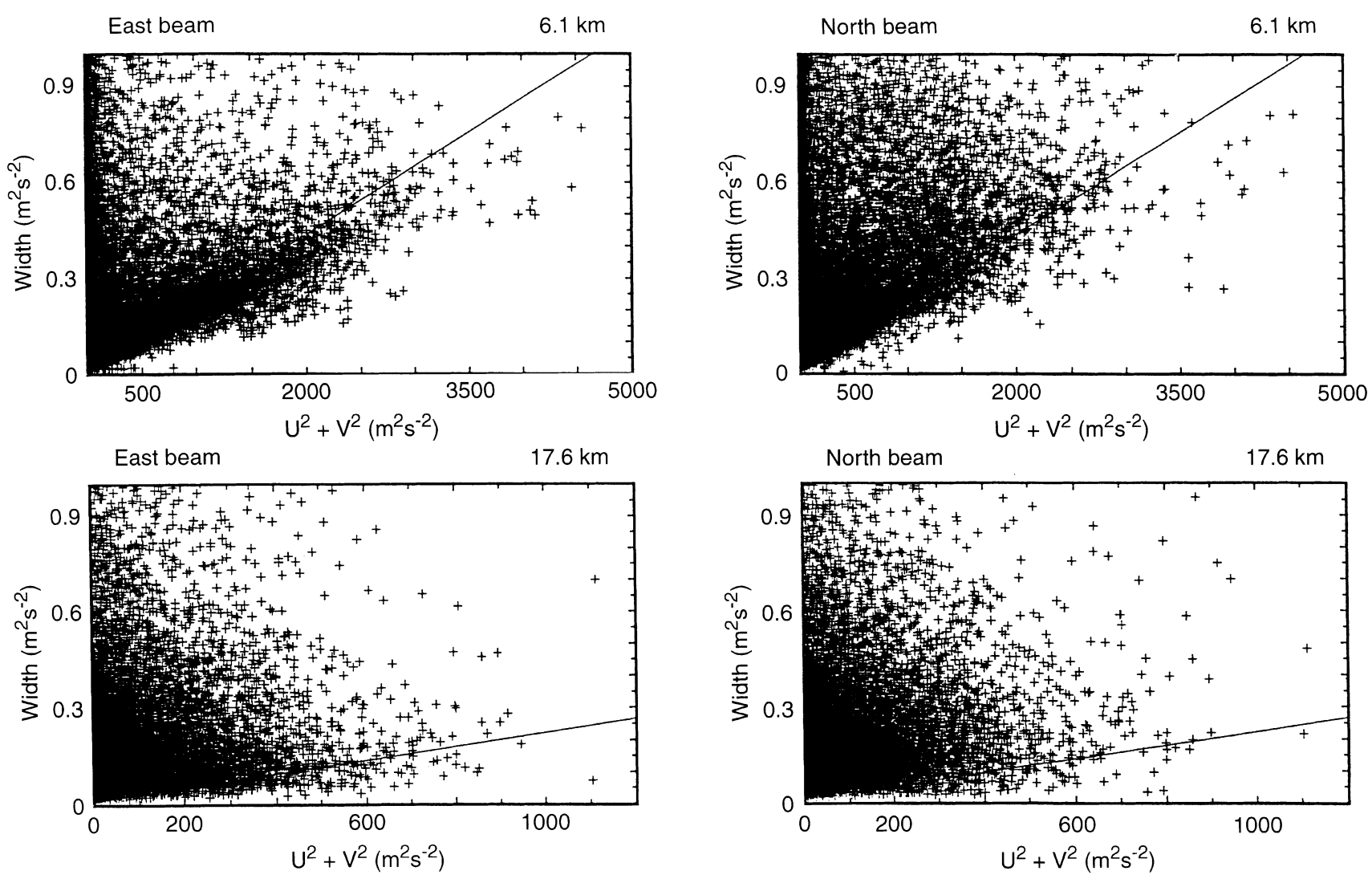

Fig. 6. Hourly means of the Doppler spectral widths observed at WSMR by the oblique beams in the (1) east-west and (2) north-south planes at 6.1 and $17.6 \mathrm{~km}$ as a function of square of the observed horizontal wind speed $(U$ and $V$ are observed zonal and meridional wind speeds, respectively). The solid line in each panel is the spectral broadening that would be expected due to beam-broadening effects alone
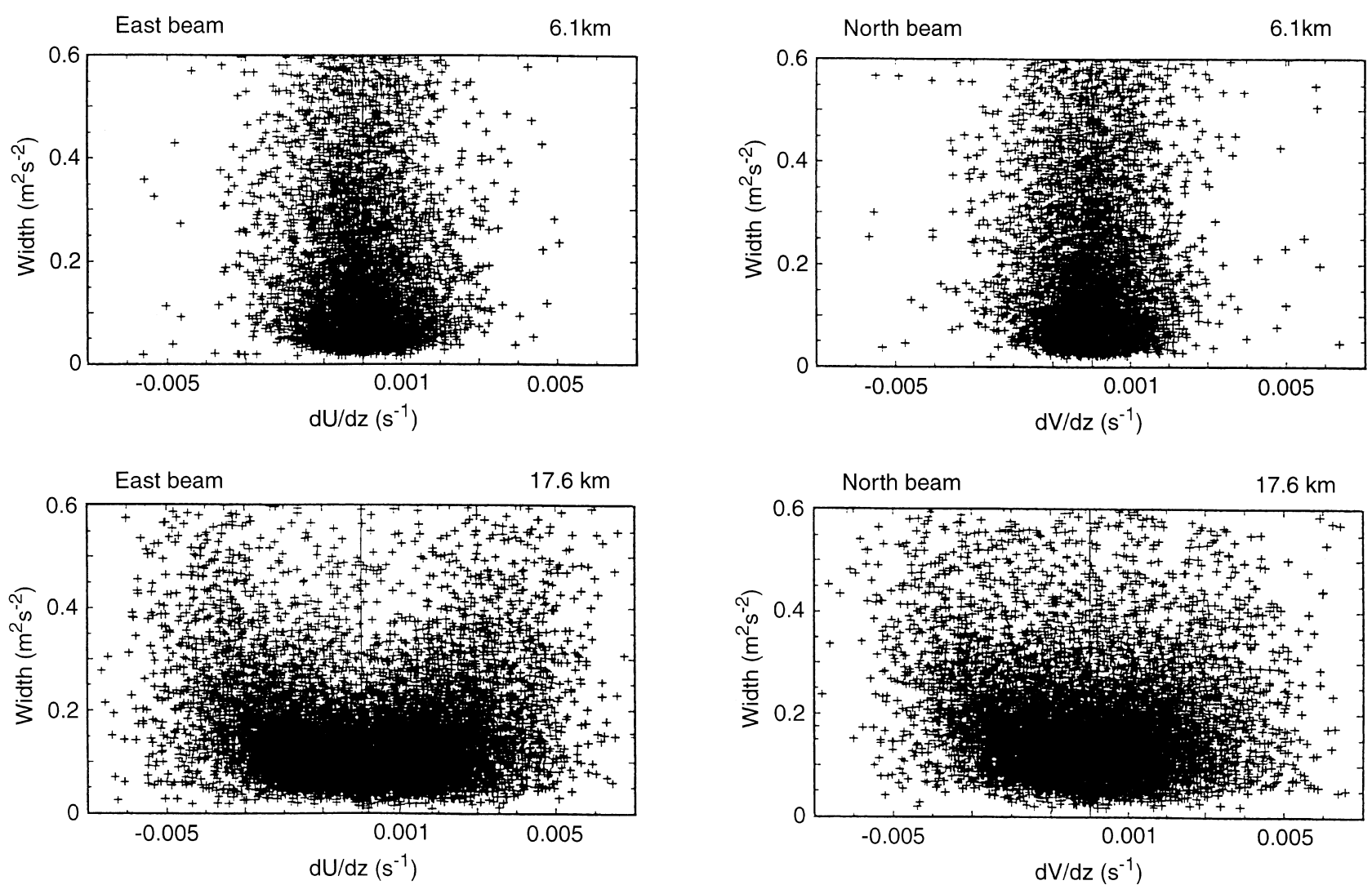

Fig. 7. Hourly means of the Doppler spectral widths observed at WSMR at 6.1 and $17.6 \mathrm{~km}$ as a function of the observed vertical shear of the horizontal wind 

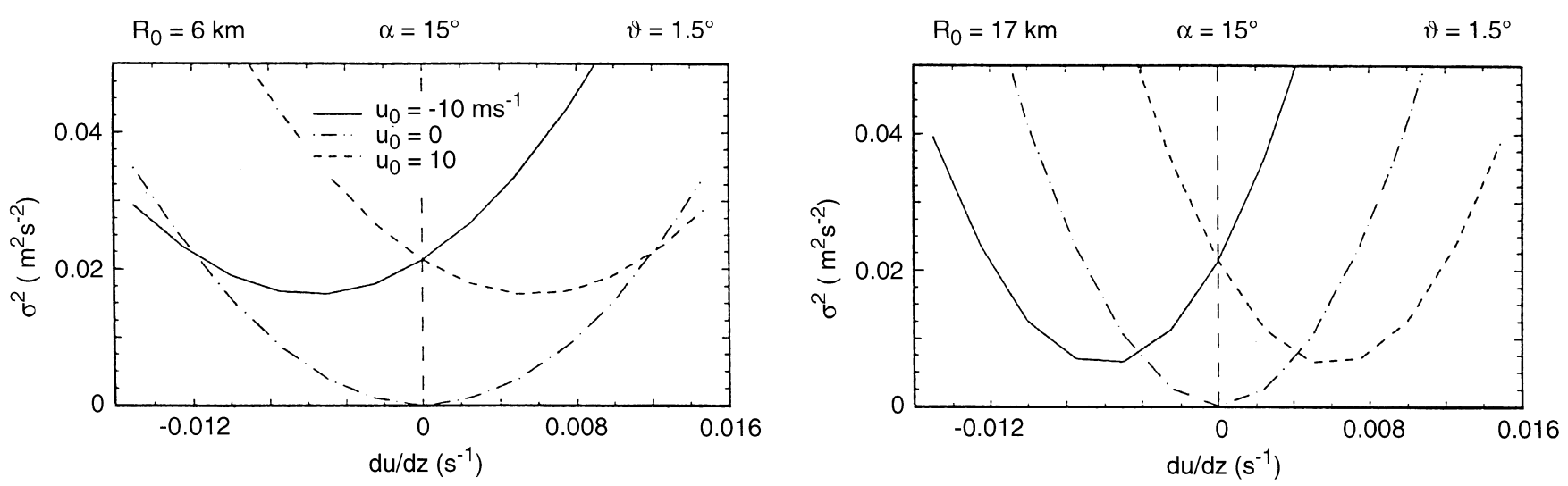

Fig. 8. As in Fig. 3, excect for typical horizontal wind speeds and for zenith distance 15 degrees and half-beamwidth 1.5 degrees at $R_{o}=6$ and $17 \mathrm{~km}$

the observed wind speed and vertical shear of the horizontal wind; since the sign of the coefficient of the second term in Eq. (15) is negative, we expect that (assuming other factors are random) the mean observed widths will be larger when the product $u_{o} \frac{\partial u}{\partial z}$ is negative. This expectation is confirmed in Table 2.

\section{Summary}

Comparisons of analyses of 1-, 2-, and 3-dimensional models of a radar beam show that, generally, it is not adequate to simply add up the separate contributions of beam- and shear-broadening from a 1-dimensional model. On the other hand, it is found that the analytical solution of a 2-dimensional beam with uniform weighting across the beam is usually a good approximation to the results of numerical integration of a 3-dimensional beam model with Gaussian weighting across the beam.

The 2-dimensional solution has a term not found in the 1-dimensional solutions and not presented in math-

Table 2. Comparisons of the mean spectral widths at $17.1 \mathrm{~km}$ at WSMR when the cases are sorted according to the algebraic sign of the product of wind speed and vertical wind shear

\begin{tabular}{cccc}
\hline & $N$ & Width (beam 1) & Width (beam 2) \\
\hline $6<\left|u_{o}\right|<10$ & & & \\
$u_{o} \partial u / \partial z<0$ & 1844 & $0.256 \pm 0.021$ & $0.244 \pm 0.015$ \\
$u_{o} \partial u / \partial z>0$ & 1541 & $0.143 \pm 0.008$ & $0.192 \pm 0.008$ \\
$\begin{array}{c}6\left|v_{o}\right|<10 \\
v_{o} \partial v / \partial z<0\end{array}$ & 501 & $0.232 \pm 0.028$ & $0.222 \pm 0.027$ \\
$\begin{array}{c}v_{o} \partial v / \partial z>0 \\
10<\left|u_{o}\right|<15\end{array}$ & 367 & $0.208 \pm 0.029$ & $0.164 \pm 0.017$ \\
$\begin{array}{c}u_{o} \partial u / \partial z<0 \\
u_{o} \partial u / \partial z>0\end{array}$ & 1510 & $0.293 \pm 0.025$ & $0.293 \pm 0.020$ \\
$10<\left|v_{o}\right|<15$ & 1014 & $0.149 \pm 0.008$ & $0.221 \pm 0.011$ \\
$v_{o} \partial v / \partial z<0$ & 127 & $0.330 \pm 0.084$ & $0.364 \pm 0.124$ \\
$v_{o} \partial v / \partial z>0$ & 108 & $0.247 \pm 0.056$ & $0.233 \pm 0.091$ \\
\hline
\end{tabular}

The standard error (twice the standard deviation divided by the square root of $N$ ) is given for each mean. The mean of the category predicted by Eq. 15 to be the largest is underlined in each case; note that the differences are much larger on beam 1 (2) when $u_{o} \partial u / \partial z\left(v_{o} \partial v / \partial z\right)$ is used to sort the cases ematical formulae in past studies. This term is due to the product of background wind speed and wind shear. This term is not always negligible; its magnitude can be as large as that of the other terms as illustrated by the sample of data in Fig. 9 where the cross-term is compared to the beam-broadening term for observations at WSMR during September-October-November at $14.1 \mathrm{~km}$. In Fig. 9, while the beam-broadening term is largest in most cases, there are a significant number of cases when the cross-term is non-negligible.

The corrections to the observed spectral width predicted by the formula developed here are consistent with observations from the VHF wind profiler at WSMR. The magnitudes of the combined effects of beam- and shear-broadening are usually less than about $0.1 \mathrm{~m}^{2} \mathrm{~s}^{-2}$ at typical heights in the troposphere and stratosphere at WSMR, as illustrated in Fig. 10, although in some cases the values of the corrections are much larger than $0.1 \mathrm{~m}^{2} \mathrm{~s}^{-2}$. (The values in Fig. 10 were computed using the total wind speed in the first term of

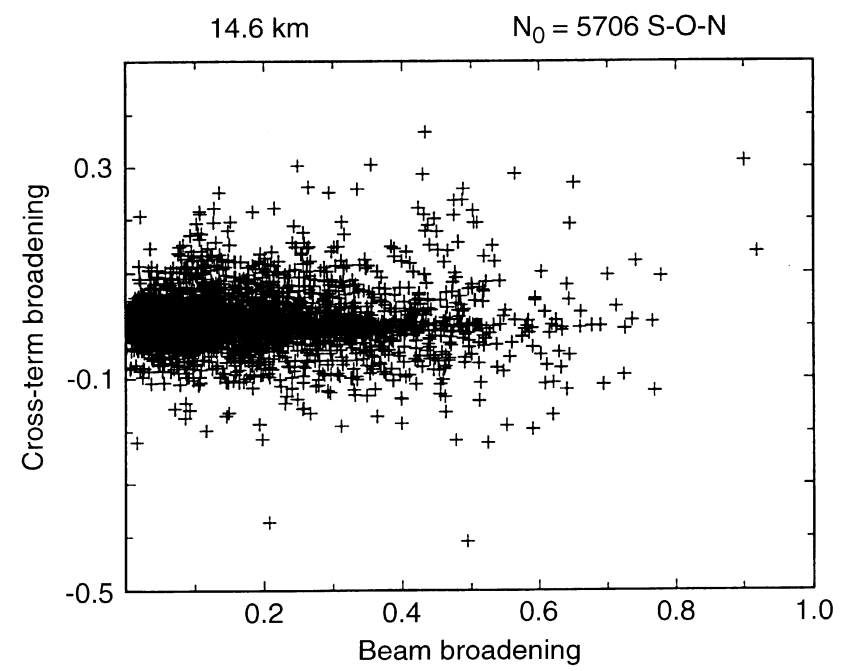

Fig. 9. Comparison of the cross-term (Term II of Eq. 16) with the beam-broadening term (Term I of Eq. 16) computed using the observed wind speeds and vertical wind shears at WSMR at $14.6 \mathrm{~km}$ during September-November 

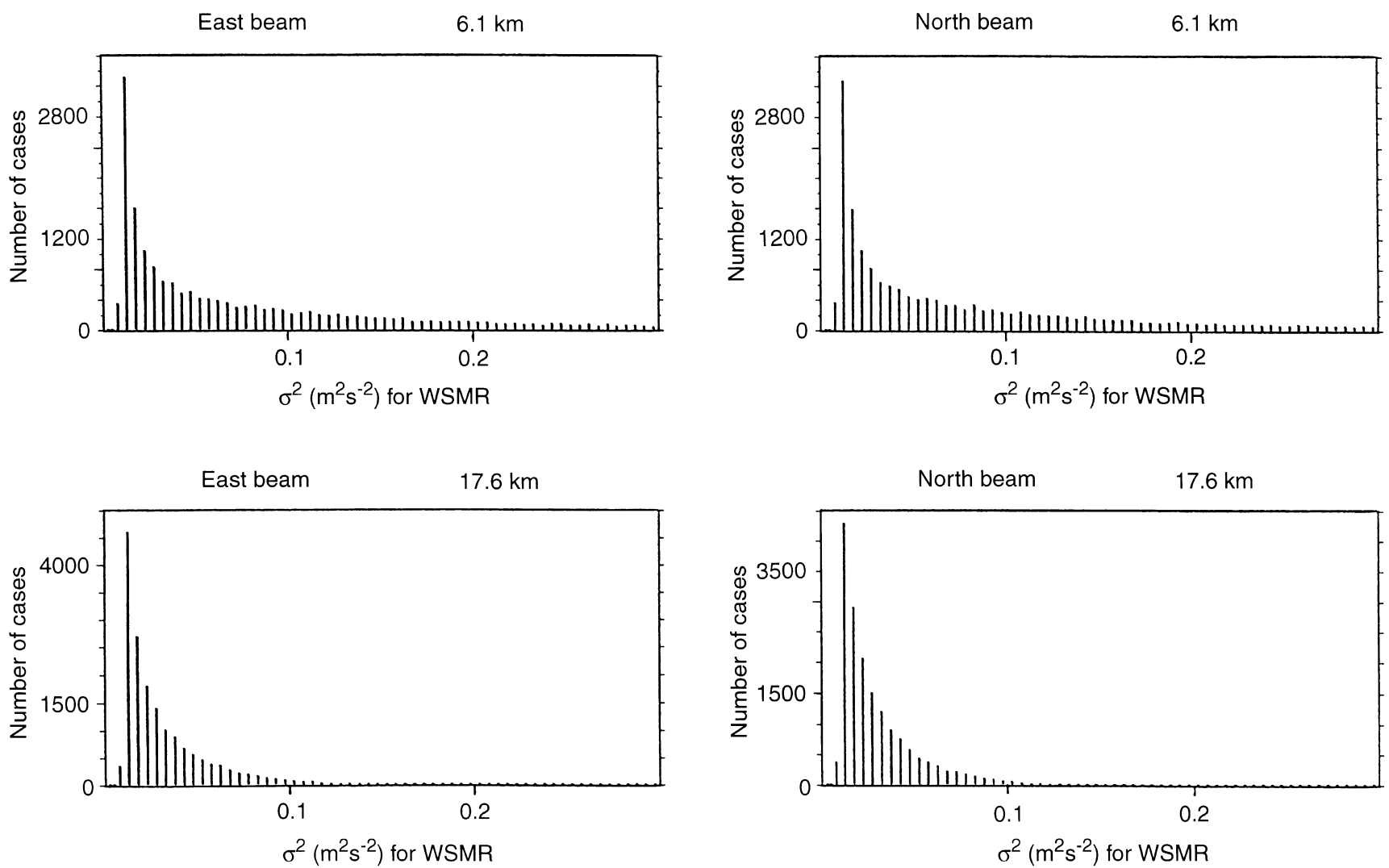

Fig. 10. Frequency distributions of the values of $\sigma^{2}$ computed with (15) using the observed wind speeds and vertical wind shears at WSMR

Eq. 15 and the wind speed and wind shear along the beam direction in the other terms). In any case, as stressed by past studies (e.g., Atlas, 1964; Hocking, 1983a, 1985; Gossard and Strauch, 1983; Gossard, 1990), the corrections to spectral width due to beambroadening and wind-shear effects must always be included in analyses of atmospheric turbulence from spectral widths.

This work was aimed at applications to wind profiler observations. However, the formula for spectral broadening is general and could be applied to observations from other radar systems such as the WSR-88D although no effort has been made here toward that application.

Acknowledgements. The author's visit in Germany was made possible by a research award from the Alexander von Humboldt Foundation. Discussions with P. Czechowsky and R. Ruster and comments by W. Hocking are gladly acknowledged. The preparation of the White Sands data was completed under NSF grant ATM-9319085.

Topical Editor F. Vial thanks W.K. Hocking and T. Tsuda for their help in evaluating this paper.

\section{References}

Atlas, D., Advances in radar meteorology. Adv. Geophys., 10, Eds., H. Landsberg and J. van Mieghem, Academic, pp. 317-478, 1964. Atlas, D., R. C. Srivastava, and P. W. Sloss, Wind shear and reflectivity gradient effects on Doppler radar spectra: II, J. Appl. Meteorol, 8, 384-388, 1969.
Cohn, S. A., Radar measurements of turbulent eddy dissipation rate in the troposphere: a comparison of techniques, J. Atmos. Ocean. Technol., 12, 85-95, 1995.

Doviak, R. J., and D. S. Zrnic, Doppler radar and weather observations, Academic, Orlando, 458pp., 1984.

Doviak, R. J., and D. S. Zrnic, Doppler radar and weather observations, 2nd ed., Academic, San Diego, 562pp., 1993.

Fukao, S., T. Sato, P. T. May, T. Tsuda, S. Kato, M. Inaba, and I. Kimura, A systematic error in MST/ST radar wind measurement induced by a finite range volume effect. 1. Observational results, Radio Sci., 23, 59-73, 1988a.

Fukao, S., M. Inaba, I. Kimura, P. T. May, T. Sato, T. Tsuda, and S. Kato, A systematic error in MST/ST radar wind measurement induced by a finite range volume effect. 2. Numerical considerations, Radio Sci., 23, 74-82, 1988b.

Fukao, S., M. D. Yamanaka, N. Ao, W. K. Hocking, T. Sato, M. Yamamoto, T. Nakamura, T. Tsuda, and S. Kato, Seasonal variability of vertical eddy diffusivity in the middle atmosphere 1. Three-year observations by the middle and upper atmosphere radar, J. Geophys. Res., 99, 18973-18987, 1994.

Hitchfeld, W., and A. S. Dennis, Turbulence in snow generating cells. Sci. Rep. MW-23, McGill University, Montreal, 31pp., 1956.

Hocking, W. K, On the extraction of atmospheric turbulence parameters from radar backscatter Doppler spectra-I. Theory, J. Atmos. Terr. Phys., 45, 89-102, 1983a.

Hocking, W. K, Mesospheric turbulence intensities measured with a HF radar at $35^{\circ}$ S-II, J. Atmos. Terr. Phys., 45, 103-114, $1983 b$.

Hocking, W. K., Measurement of turbulent energy dissipation rates in the middle atmosphere by radar techniques: a review, Radio Sci., 20, 1403-1422., 1985.

Hocking, W. K. Observation and measurement of turbulence in the middle atmosphere with a VHF radar, J. Atmos. Terr. Phys., 48, 655-670, 1986. 
Hocking, W. K. Two years of continuous measurements of turbulence parameters in the upper mesosphere and lower thermosphere made with a 2-MHz radar, J. Geophys. Res., 93, 2475-2491, 1988.

Gossard, E. E, Radar research on the atmospheric boundary layer, in Ed. D. Atlas Radar in meteorology, pp. 477-527., 1990.

Gossard, E. E., and R. G. Strauch, Radar observations of clear air and clouds, Elsevier, 280 pp, 1983.

Murphy, D. J., W. K. Hocking, and D. C. Fritts, An assessment of the effect of gravity waves on the width of radar Doppler spectra, J. Atmos. Terr. Phys., 56, 17-29, 1994.

Nastrom, G. D., and F. D. Eaton, The coupling of gravity waves and turbulence at White Sands, New Mexico, from VHF radar observations, J. Appl. Meteorol, 32, 81-87, 1993.
Nastrom, G. D., and F. D. Eaton, Variations of winds and turbulence seen by the $50 \mathrm{MHz}$ radar at White Sands Missile Range, NM, J. Appl. Meteoro., 34, 2135-2148, 1995.

Nastrom, G. D., and F. D. Eaton, Turbulence eddy dissipation rates from radar observations at 5-20 km at White Sands Missile Range, NM, J. Geophys. Res., in press 1997.

Sirmans, D., and R. J. Doviak, Meteorological radar signal intensity estimation, NOAA Tech. Mem. ERL NSSL 64, 80 pp., 1973.

Sloss, P. W., and D. Atlas, Wind shear and reflectivity gradient effects on Doppler radar spectra, J. Atmos. Sci., 25, 1080-1089, 1968. 